\title{
Pathway dependence in the fuel-driven dissipative self-assembly of nanoparticles.
}

\author{
Raphael K. Grötsch ${ }^{1}$, Caren Wanzke ${ }^{1}$, Maximilian Speckbacher ${ }^{2}$, Arzu Angi ${ }^{3}$, Bernhard Rieger ${ }^{3}$ and Job \\ Boekhoven $^{* 1,4}$ \\ 1 Department of Chemistry, Technical University of Munich, Lichtenbergstraße 4, 85748 Garching (Germany). \\ 2 Molecular Electronics, Department of Electrical and Computer Engineering, Technical University of Munich, \\ Theresienstraße 90, 80333 Munich (Germany). \\ 3 WACKER-Lehrstuhl für Makromolekulare Chemie, Technical University of Munich, Lichtenbergstraße 4, 85748 Garching \\ (Germany) and Catalysis Research Center, Ernst-Otto-Fischer-Straße 1, 85748 Garching (Germany). \\ 4 Institute for Advanced Study, Technical University of Munich, Lichtenbergstraße 4, 85748 Garching (Germany).
}




\section{Supporting Information}

\section{Table of Content}

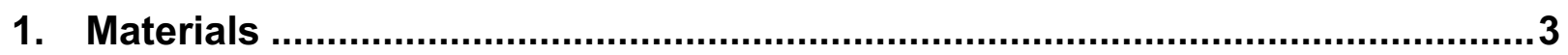

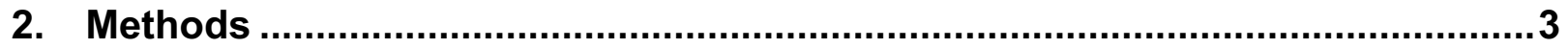

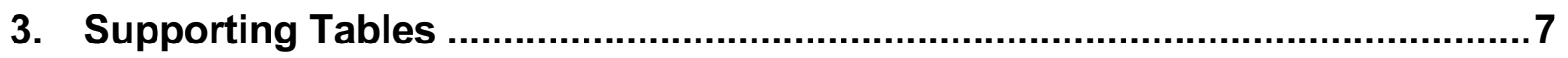

Table SI1. Dynamic light scattering - Precursors of gold and iron oxide

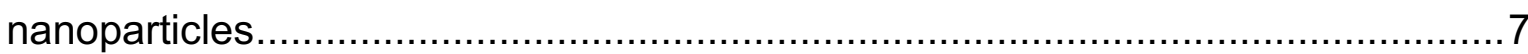

Table SI2. DLS - Pathway complexity of gold and iron oxide nanoparticles ...........8

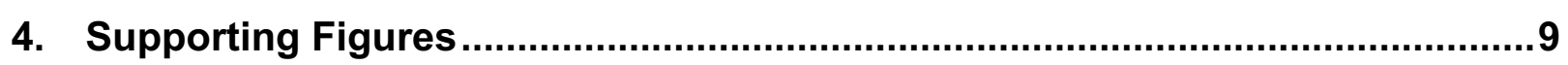

Figure SI1. Cryogenic-transmission electron microscopy of gold and iron oxide

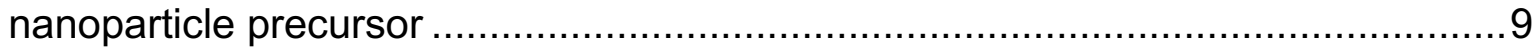

Figure SI2. Titration of gold and iron oxide nanoparticles................................ 10 Figure SI3. FTIR spectroscopy after addition of 0.3 equivalents of EDC to the gold nanoparticles.

Figure SI4. DLS - diameter of gold nanoparticles ........................................12

Figure SI5. DLS - diameter of gold nanoparticles ..........................................13

Figure SI6. Supporting Scanning Electron Microscopy ...................................14

Figure SI7. Time-lapsed photography for the addition of 0.8 equivalents of EDC to gold nanoparticles

Figure SI8. FTIR of $0.3,0.5$ and 0.8 equivalents of EDC addition to gold nanoparticles.

Figure SI9. DLS of $0.3-2$ equivalents of EDC addition to gold nanoparticles......17

Figure SI10. Supporting Transmission Electron Microscopy 18

Figure SI11. FTIR data for the addition of 0.4 and 0.8 equivalents of EDC to the iron oxide nanoparticles

5. References. 


\section{Materials}

Tetrachloroauric (III) acid trihydrate and trisodium-citrate dihydrate were purchased from Carl Roth. Iron oxide (II,II) magnetic nanoparticles solution with $10 \mathrm{~nm}$ in diameter and carboxylic acids as reactive groups on the surface (measured diameter by cryo-TEM = $15 \mathrm{~nm}$; SPH-10 organic layer: Monolayer of oleic acid bound to the iron oxide and coassembled on to the hydrophobic alkyl chain hydrolyzed polymaleic anhydride-alt-1octadecene on the surface, for a detailed description of the synthesis see Nie et. $\mathrm{al}^{1}$ ), sodium 3-mercaptopropionic acid, N-Hydroxysuccinimide (NHS), 1-ethyl-3-(3dimethylaminopropyl)carbodiimide (EDC), and 2-(N-morpholino) ethanesulfonic acid (MES) were purchased from Sigma-Aldrich. All chemicals were used without further purification. Water was obtained from a Millipore water purifier system.

\section{Methods}

\section{Synthesis of gold NP}

The synthesis followed a modified Turkevich protocol. ${ }^{2}$ A tetrachloroauric (III) acid solution ( $\mathrm{HAuCl} 4 \cdot 3 \mathrm{H} 2 \mathrm{O}, 5,8 \mathrm{mmol} / \mathrm{l}, 25 \mathrm{ml}$ ) was poured into refluxing water $(250 \mathrm{ml}$ ). After the solution boiled again, a solution of sodium 3-mercaptopropionic acid (0,39 $\mathrm{mmol} / \mathrm{l}, 25 \mathrm{ml})$ and trisodium citrate dihydrate $(2,0 \mathrm{wt} \%)$ was injected. Heating for 1,5 hours yielded a pale red solution. The solution was slowly cooled to room temperature.

\section{Determination of the concentration of surface bonded carboxylic acid groups}

A fraction of the gold nanoparticles or iron oxide nanoparticles was dispersed in water and the $\mathrm{pH}$ was set to $\mathrm{pH} 10.5$ by addition of $\mathrm{NaOH}$ (conc.). It was titrated against $\mathrm{HCl}$ $(0.01 \mathrm{M})$ to determine the pKa and the number of carboxylates on the nanoparticle surface in the sample (See Figure SI1).

\section{Average number of ligands per gold nanoparticle}

Based on the TEM, we find the particles have a diameter of $3.5 \mathrm{~nm}$ (see below). That diameter corresponds to an average surface area per particle $38.5 \mathrm{~nm}^{2}$. The surface area a thiol ligand occupies on a gold nanoparticle has been found to be $0.16 \mathrm{~nm}^{2}$. 
From these numbers, we can calculate that on average each particles contains roughly 250 molecules of mercaptopropionic acid.

\section{Reaction conditions for the chemical reaction cycle}

Gold nanoparticles were dispersed in MES buffer $(0.02 \mathrm{M}, \mathrm{pH}=6.4)$ with $25 \mathrm{mM}$ NHS to yield a $0.25 \mathrm{mM}$ solution of carboxylates surface groups. Iron oxide nanoparticles were dispersed in MES buffer $(0.2 \mathrm{M}, \mathrm{pH}=6.5)$ with $20 \mathrm{mM} \mathrm{NHS}$ to yield a $5 \mathrm{mM}$ solution of carboxylates surface groups.

In order to start the cycle, EDC was added from a high-concentration stock solution. The solution was pipetted a few times to induce mixing and allowed to stand without stirring. We noticed that stirring increased the tendency of the particles to precipitate in response to EDC.

\section{Dynamic light scattering (DLS).}

DLS measurements were performed using a Malvern Zetasizer Nano ZS with a laser wavelength of $633 \mathrm{~nm}$. The nanoparticles solutions were measured using disposable cuvettes (PS). Each measurement consisted of 6 acquisitions with an acquisition time of $20 \mathrm{~s}$.

\section{Transmission electron microscopy (TEM)}

Cryo-TEM imaging was performed on a Tecnai Spirit microscope (FEI/Thermo Fisher) operating at $120 \mathrm{kV}$ or a Jeol JEM-1400 plus operating at $120 \mathrm{kV}$. The grids (C-Flat R1.2/1.3, 400 mesh, Cu or Quantifoil R2/2 on Cu-grid 400 mesh were used) were freshly glow-discharged for 90 seconds with $50 \mathrm{~mA}$ prior to use. Preparation of the grids was performed in a FEl/Thermo Fisher Vitrobot at $25^{\circ} \mathrm{C}$ with the relative humidity set to $100 \%$. The sample was incubated for 30 seconds on the grid, blotted for 2 seconds (blotting force set to -1) and then directly plunged into liquid ethane that was pre-cooled by liquid nitrogen. The cryo-TEM grids were transferred and stored in liquid nitrogen, and when needed, placed into a Gatan cryo-transfer-specimen holder to insert into the microscope. The specimen temperature was maintained at $-170{ }^{\circ} \mathrm{C}$ during the data collection. The images were recorded in a low-dose mode on a CCD camera. 
TEM imaging was performed on a Tecnai Spirit microscope (FEI/Thermo Fisher) operating at $120 \mathrm{kV}$. The Cu400 TEM grids were collodion-supported, carbon coated and freshly glow-discharged for 45 seconds with $35 \mathrm{~mA}$ prior to use. The sample was incubated for 45 seconds on the grid, blotted for 1 second and dried for $15 \mathrm{~min}$. The images were recorded in a low-dose mode on a CCD camera.

\section{Scanning electron microscopy (SEM)}

SEM imaging was performed on a Zeiss NVision 40 operating at an electron high tension of $5 \mathrm{kV}$. Samples for SEM were prepared as described above. 24 hours after the EDC addition, the buffer was slowly exchanged with methanol. The clusters in methanol were transferred on a Si-wafer.

\section{Fourier-transform infrared spectroscopy (FTIR).}

First, the nanoparticles were isolated by acidifying the reaction solution at the indicated times during or before the cycle. Then the aggregated nanoparticles were centrifuged, the supernatant was removed and the NCs were redispersed in ethanol and acidified again. The centrifugation and dispersion steps were performed three times to obtain a pellet of precipitated particles. The pellet of nanoparticles were redispersed in $30 \mu \mathrm{L}$ of ethanol and then dried on the measurement-crystal of the Bruker Vertex 70 FTIR using a platinum ATR from Bruker.

\section{High pressure liquid chromatography (HPLC)}

The kinetics of the chemical reaction networks were monitored over time by means of analytical HPLC (HPLC, Thermofisher Dionex Ultimate 3000, Hypersil Gold $250 \times 4.8$ $\mathrm{mm}, 25^{\circ} \mathrm{C}$ ). A $400 \mu \mathrm{L}$ sample was prepared as described above, filtered with a $25 \mathrm{~mm}$ syringe filter ( $200 \mathrm{~nm}$ polyethersulfone) and placed into a screw cap HPLC vial. Every 10 minutes, samples of the freshly filtered solutions were directly injected without further dilution, and all compounds involved were separated using a linear gradient water: ACN from 100:0 to 70:30. Calibration curves for EDC $(\lambda=220 \mathrm{~nm})$ were performed in duplicate in order to quantify the compound over time. 
Time-lapse photography. In a $1 \mathrm{ml} \mathrm{HPLC}$-vial, 600 - $750 \mu \mathrm{L}$ of NP solution were imaged with a high-definition camera. Time-lapse software was programmed to image the sample in intervals from 2 to $30 \mathrm{~min}$. 


\section{Supporting Tables}

Table SI1. Dynamic light scattering - Precursors of gold and iron oxide nanoparticles

Table SI1. The hydrodynamic diameter (d), derived count rate (DCR) and polydispersity index (PDI) data of gold nanoparticles (NP) and iron oxide nanoparticles as obtained by dynamic light scattering (DLS) in MES (2-(N-morpholino) ethanesulfonic acid) buffered aqueous solution.

\begin{tabular}{l|lll|lll|}
\multicolumn{3}{c}{ Gold NP } & \multicolumn{3}{c}{ Iron oxide NP } \\
\hline \hline & $\begin{array}{l}\text { d } \\
\text { (nm) }\end{array}$ & $\begin{array}{l}\text { DCR } \\
(\mathrm{mcps})\end{array}$ & $\begin{array}{l}\text { PDI } \\
(\%)\end{array}$ & $\begin{array}{l}\mathrm{d} \\
(\mathrm{nm})\end{array}$ & $\begin{array}{l}\text { DCR } \\
(\mathrm{mcps})\end{array}$ & $\begin{array}{l}\text { PDI } \\
(\%)\end{array}$ \\
\hline \hline & 13.1 & 1.90 & 63 & 27.9 & 14.6 & 27 \\
& 12.7 & 1.91 & 56 & 18.4 & 14.7 & 26 \\
& 12.1 & 1.90 & 56 & 20.5 & 14.7 & 26 \\
& 12.2 & 1.89 & 63 & 35.2 & 14.8 & 27 \\
& 12.0 & 1.89 & 56 & 31.3 & 14.7 & 27 \\
& 13.3 & 1.90 & 55 & 19.6 & 14.6 & 28 \\
& 12.9 & 1.88 & 62 & 37.1 & 14.7 & 28 \\
& 12.7 & 1.89 & 56 & 28.6 & 14.7 & 27 \\
& 12.2 & 1.87 & 61 & 32.5 & 14.6 & 27 \\
& 13.2 & 1.89 & 55 & 30.6 & 14.7 & 29 \\
\hline Mean & $12.6 \pm 0.5$ & $1.89 \pm 0.01$ & $58 \pm 4$ & $28 \pm 7$ & $14.7 \pm 0.1$ & $27 \pm 1$ \\
\hline \hline
\end{tabular}




\section{Table SI2. DLS - Pathway complexity of gold and iron oxide nanoparticles}

Table SI2. The hydrodynamic diameter (d), derived count rate (DCR) data of the gold and iron oxide nanoparticles obtained after the multiple addition of EDC to precursor solutions ( $3 \times 0.3$ equivalents every 24 h, $2 \times 0.45$ equivalents every $36 h, 1 \times 0.9$ equivalents for the gold nanoparticles; $5 \times 0.4$ equivalents every 48 h, 1x 2.0 equivalents for iron oxide nanoparticles). The samples were measured after 3 or 10 days, respectively.

\begin{tabular}{l|ll|ll}
\multicolumn{2}{c}{ Gold NP } & \multicolumn{2}{c}{ Iron oxide NP } \\
\hline \hline & $\mathrm{d} / \mathrm{nm}$ & $\mathrm{DCR} / \mathrm{mcps}$ & $\mathrm{d} / \mathrm{nm}$ & DCR /mcps \\
\hline Precursor & $12.6 \pm 0.5$ & $1.89 \pm 0.01$ & $28 \pm 7$ & $14.7 \pm 0.1$ \\
\hline 3x 0.3 equivalents; 3d & $14 \pm 1$ & $2.39 \pm 0.01$ & & \\
$2 \times 0.45$ equivalents; 3d & $15 \pm 1$ & $2.15 \pm 0.01$ & & \\
1x 0.9 equivalents; 3d & $20 \pm 4$ & $0.53 \pm 28$ & & \\
\hline 5x 0.4 equivalents;10 d & & & $27 \pm 1$ & $14.8 \pm 0.1$ \\
1x 2.0 equivalents;10 d & & & $-^{*}$ & $-^{*}$ \\
\hline \hline
\end{tabular}

* Due to the precipitation, insufficient nanoparticles were in the solution to measure reliable data for the iron oxide NP sample 10 days after the addition of 2.0 equivalents of EDC via DLS. 


\section{Supporting Figures}

Figure SI1. Cryogenic-transmission electron microscopy of gold and iron oxide nanoparticle precursor

The cryogenic-transmission electron microscopy (cryo-TEM) micrographs of the precursor solutions are used for the size distribution analyses. Following the guidelines of Murphy and Buriak and Scott's rule for the bin width, ${ }^{4,5}$ we measured 300 nanoparticles and depicted the results in a histogram.
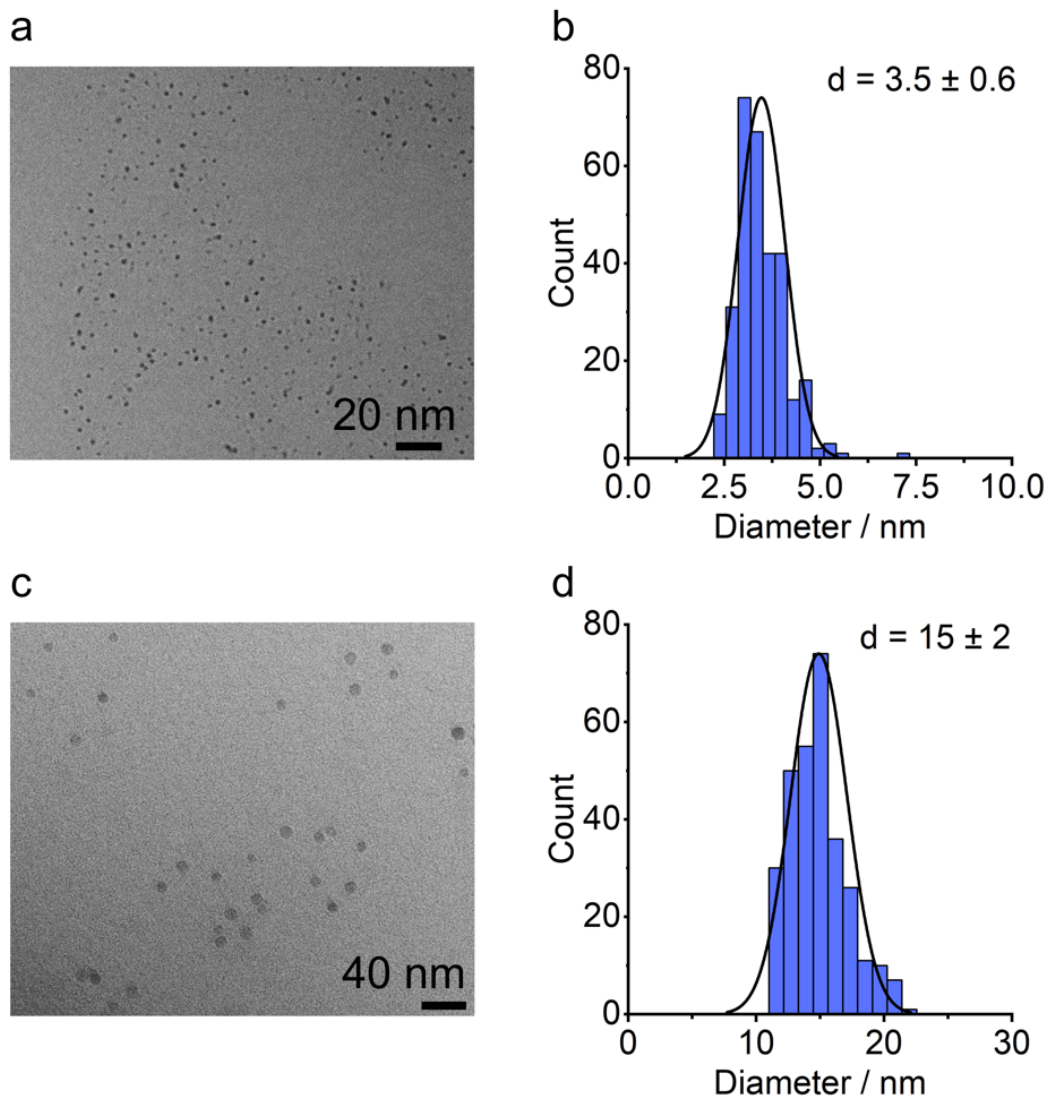

Figure SI1. Cryo-TEM micrographs of a) gold and c) iron oxide nanoparticles in MES buffer. Histogram of the b) gold and $\mathbf{c}$ ) iron oxide nanoparticle diameters size distribution. 
Figure SI2. Titration of gold and iron oxide nanoparticles

a

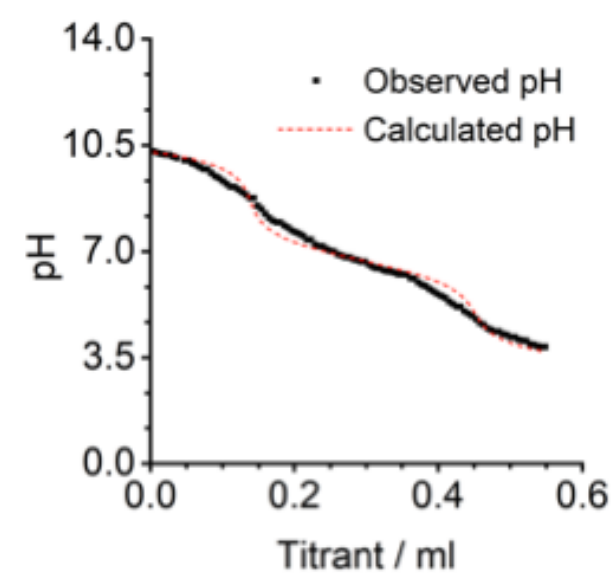

b

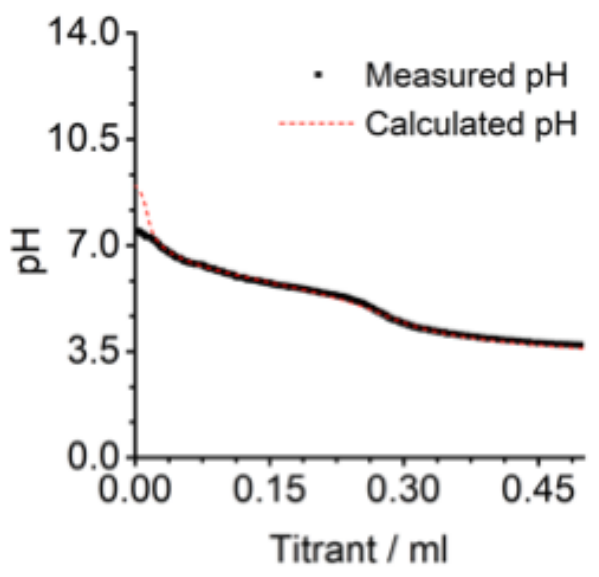

Figure SI2. Plot of the $\mathrm{pH}$ value as a function of the titrant $(0.01 \mathrm{M} \mathrm{HCl})$ for a) gold nanoparticles and b) iron oxide nanoparticles. The black curves indicate the measured values, whereas the red markers indicate the fitted values by Hyperquad 2008. Based on the fit, we calculated the pKa of the surface bound acids to a) 6.7 and b) 5.8 . 
Figure SI3. FTIR spectroscopy after addition of 0.3 equivalents of EDC to the gold nanoparticles

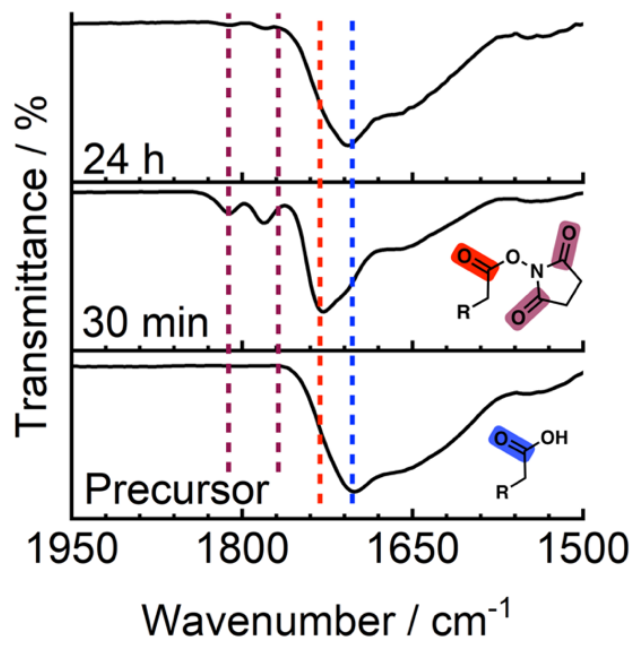

Figure SI3. Fourier-transformed Infrared (FTIR) diagram of the precursor, 30 minutes and 24 hours after the addition of 0.3 equivalents of EDC to a solution of the gold nanoparticles and NHS. Dashed lines from purple to blue mark the peaks for 1811, 1782, 1734 and $1705 \mathrm{~cm}^{-1}$, respectively. 
Figure SI4. DLS - diameter of gold nanoparticles

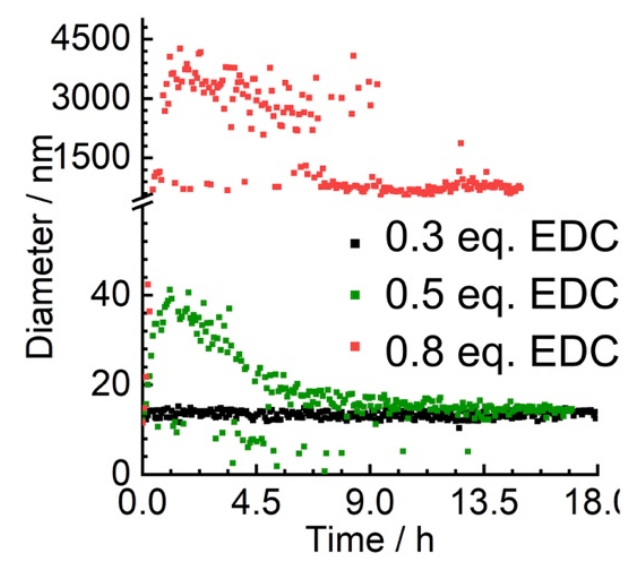

Figure S14. Plot of the diameter of the particles and assemblies as a function of time as measured by DLS. The size changes were induced by the EDC addition. 
Figure SI5. DLS - diameter of gold nanoparticles
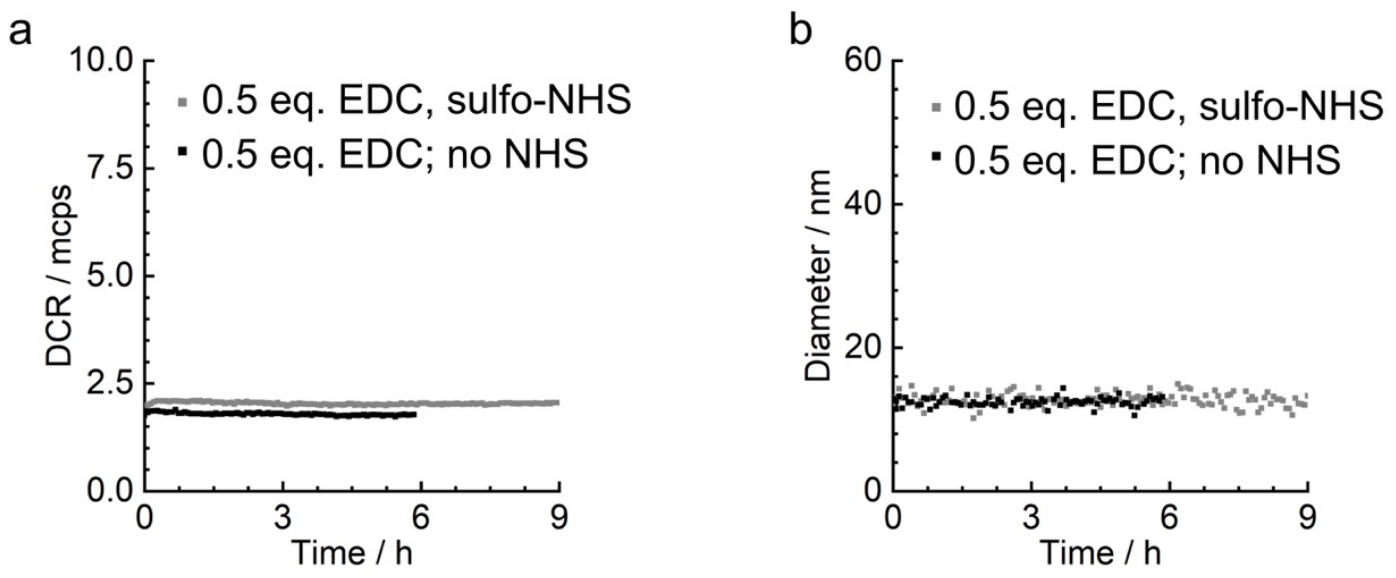

Figure SI5. Plot of $a$ ) the scattering rate and $b$ ) the diameter of the gold nanoparticles as a function of time measured by DLS. In the first experiment sulfo-NHs, which carries a negative charge, replaced NHS. In the second experiment no NHS at all was added. 
Figure SI6. Supporting Scanning Electron Microscopy

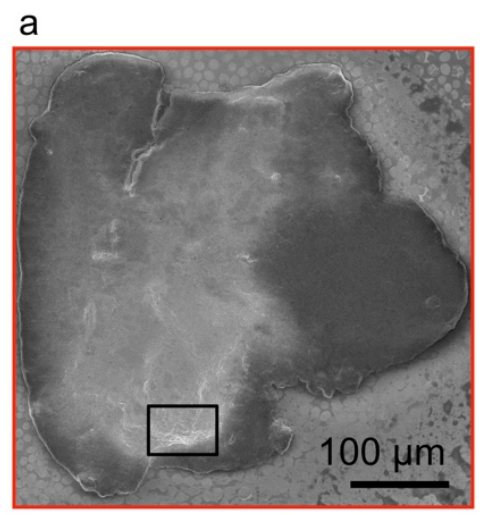

b
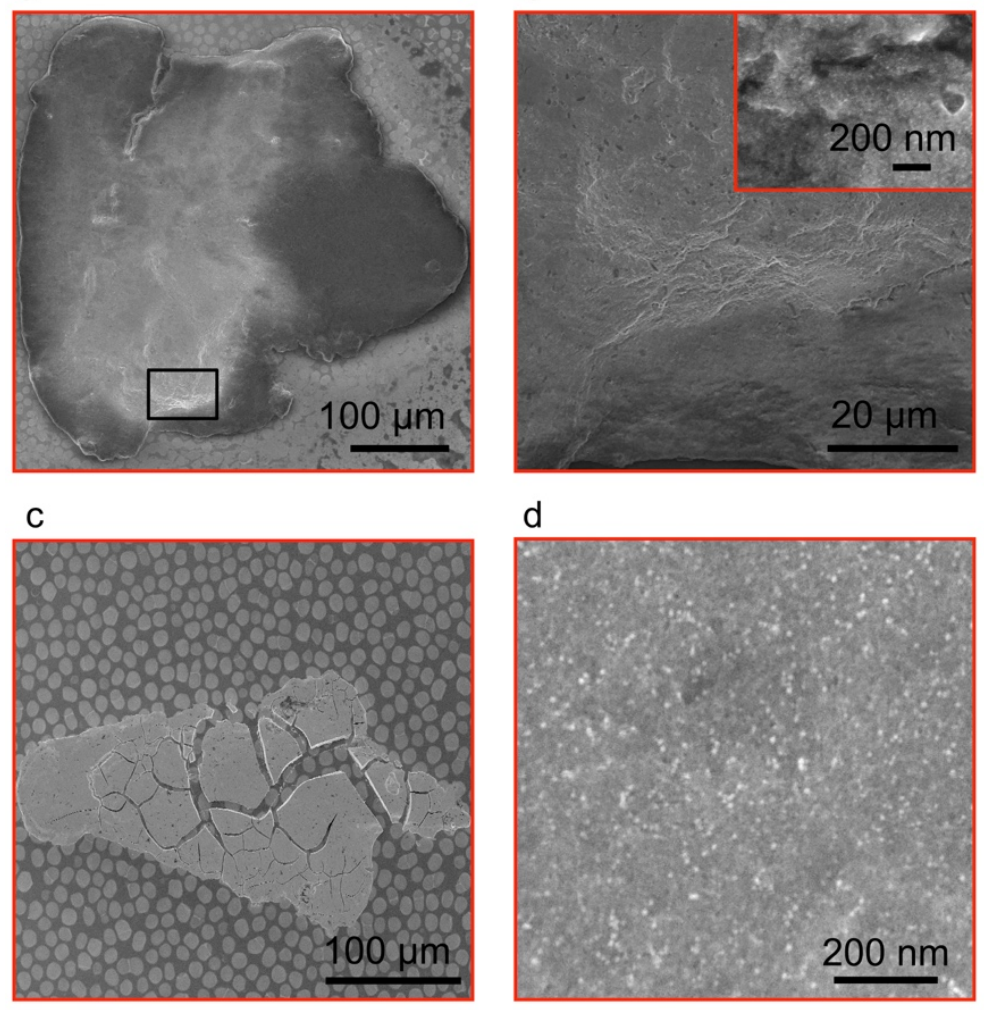

d

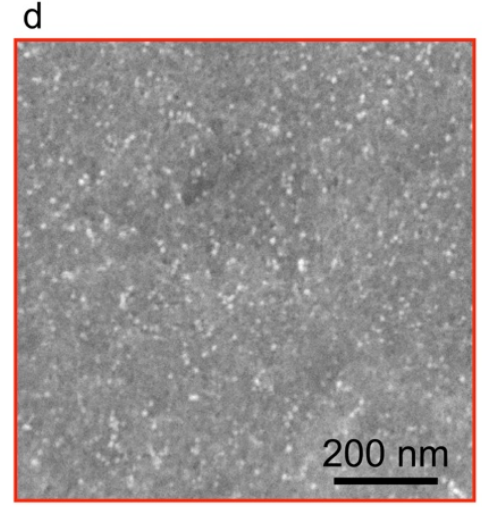

Figure SI6. a - d) Scanning electron microscopy (SEM) micrographs of clusters of the precipitate that formed after the addition of 0.8 equivalents of EDC. 
Figure S17. Time-lapsed photography for the addition of 0.8 equivalents of EDC to gold nanoparticles

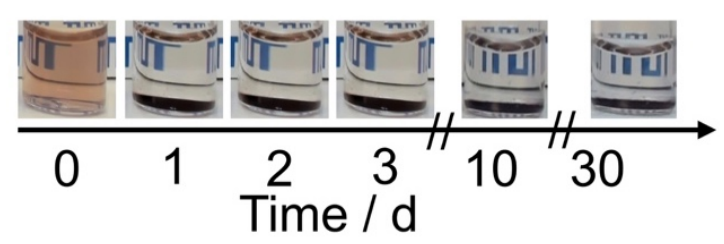

Figure SI7. Time-lapse photographs of a precursor solution after the addition of 0.8 equivalents of EDC. 
Figure SI8. FTIR of $0.3,0.5$ and 0.8 equivalents of EDC addition to gold nanoparticles

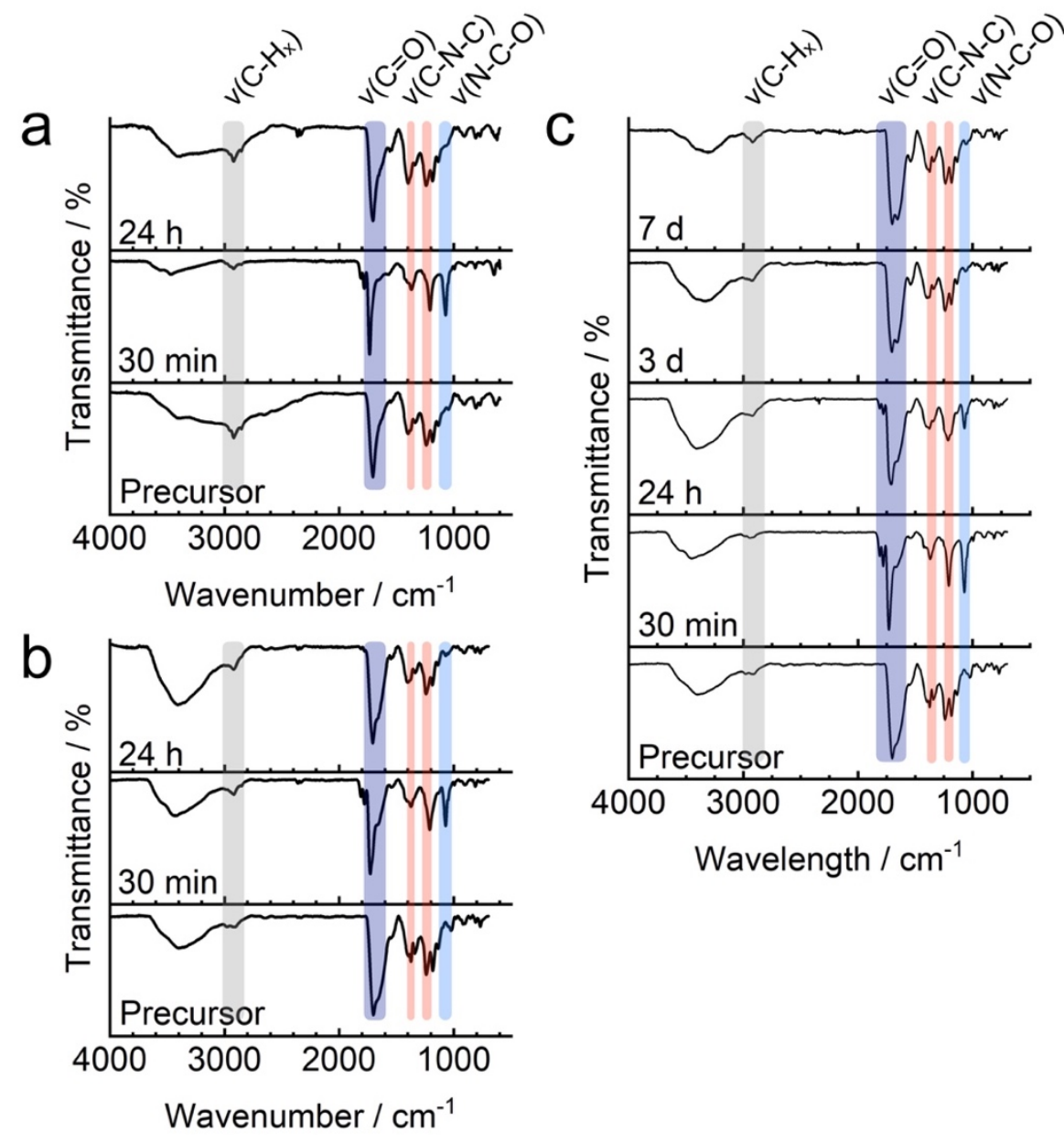

Figure SI8. FTIR spectra of gold nanoparticles. Three timepoints depicted for the addition of a) 0.5 b) 0.3 equivalents of EDC and five timepoints for the addition of c) 0.8 equivalents of EDC. Broad O-H band at $3000-3500 \mathrm{~cm}^{-1}$, propionic acid functionalized gold nanoparticles alkyl peaks at $2900 \mathrm{~cm}^{-1}\left(\mathrm{C}-\mathrm{H}_{2} / \mathrm{C}-\mathrm{H}_{3}\right)$, Carbonyl peak at $1705 \mathrm{~cm}^{-1}$ $(\mathrm{C}=\mathrm{O})$. Additional NHS-ester peaks at 1814,1784 , and $1738 \mathrm{~cm}^{-1}$ appear, originating from the carbonyl groups of the NHS ester. In addition, characteristic signals of the succinimide cycle were observed at $1370 \mathrm{~cm}^{-1} \mathrm{vs}(\mathrm{C}-\mathrm{N}-\mathrm{C}), 1206 \mathrm{~cm}^{-1} \mathrm{vs}(\mathrm{C}-\mathrm{N}-\mathrm{C})$, and $1065 \mathrm{~cm}^{-1}$ vs(N-C-O). c) FTIR spectrum 24 hours or 7 days after EDC addition. The signals of NCs with propionic acid surface groups have reappeared. 
Figure SI9. DLS of $0.3-2$ equivalents of EDC addition to gold nanoparticles

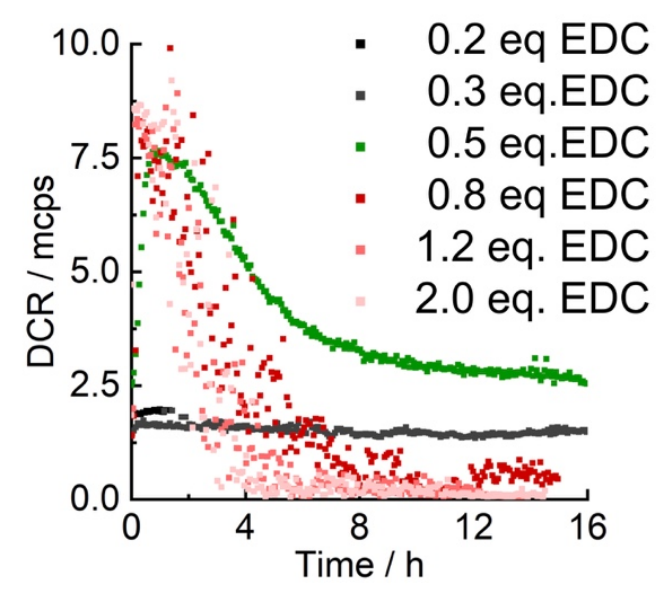

Figure S19. DLS diagram of the diameter as a function of time. Size changes induced by EDC addition (black 0.2, grey 0.3, green 0.5 and red $0.8,1.2,2.0$ equivalents of EDC). 
Figure S110. Supporting Transmission Electron Microscopy
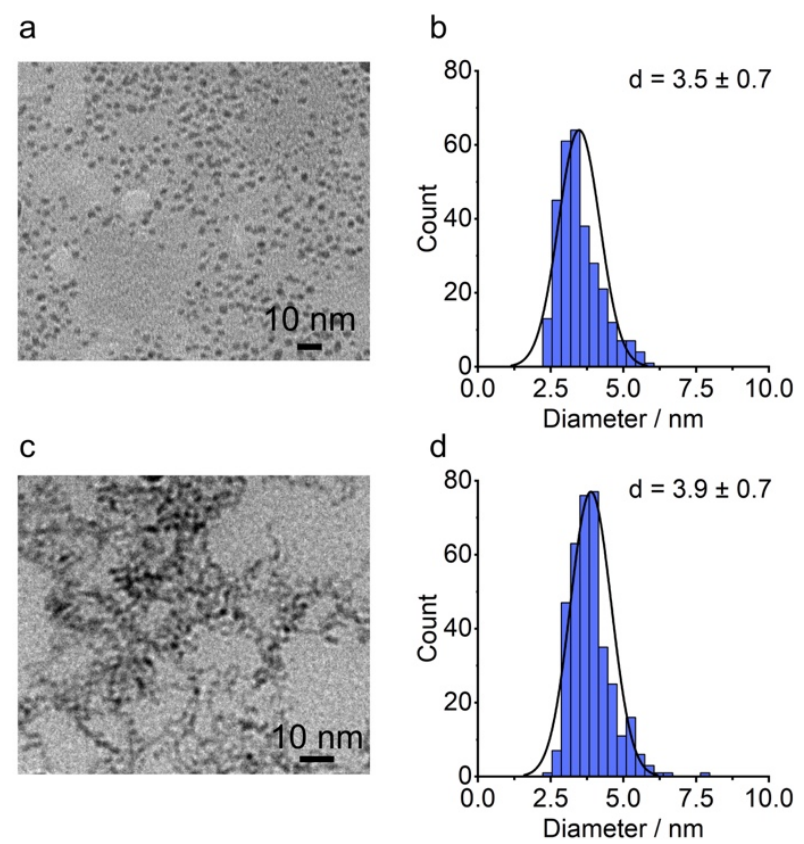

Figure SI10. TEM micrographs of gold nanoparticles a) in MES buffer and c) $24 \mathrm{~h}$ after the addition of 0.8 eq. of EDC. Histogram of gold nanoparticles diameter size distribution b) in MES buffer and d) $24 \mathrm{~h}$ after the addition of 0.8 eq. of EDC. 


\section{Figure SI11. FTIR data for the addition of 0.4 and 0.8 equivalents of EDC to the iron oxide nanoparticles}

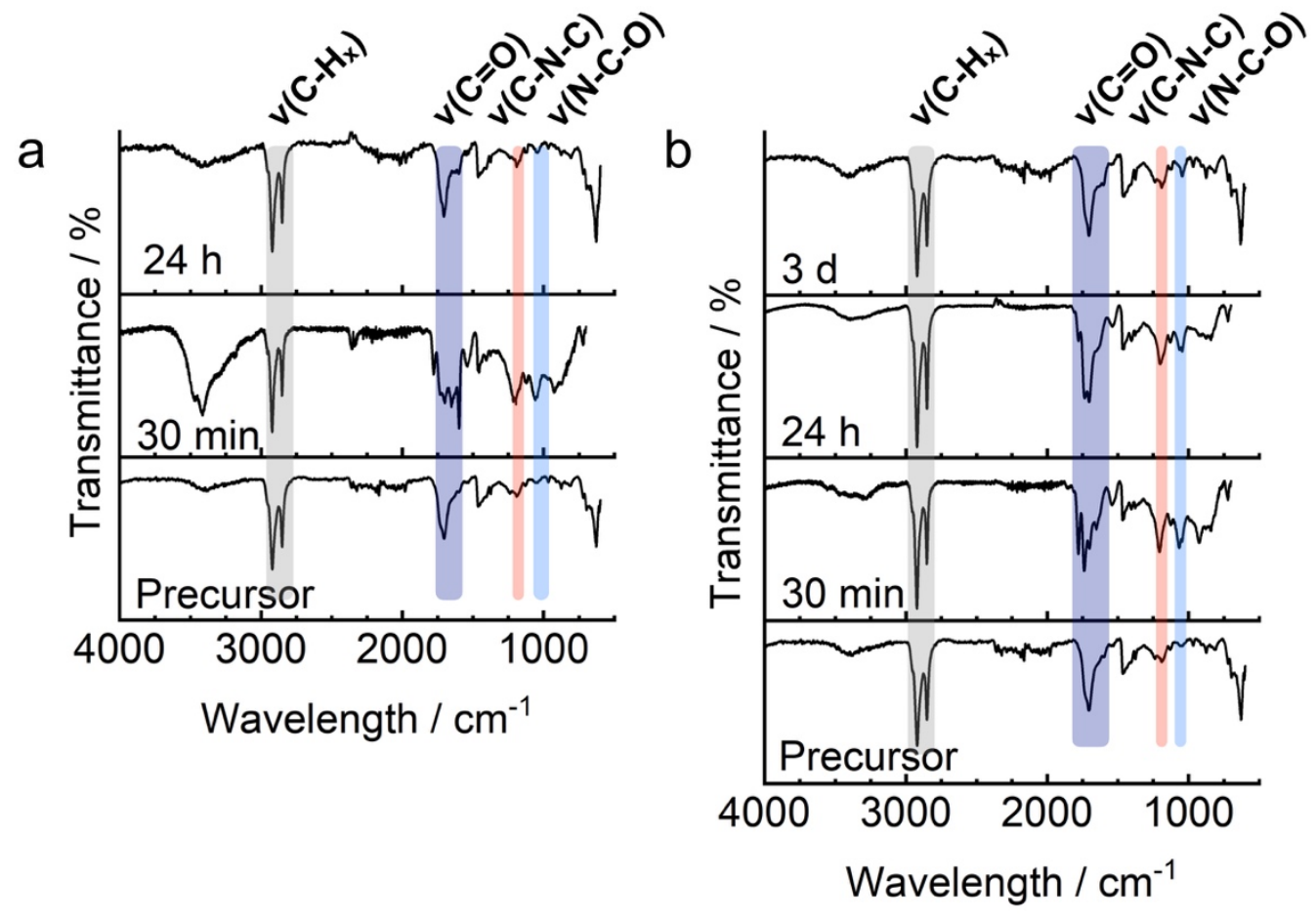

Figure SI11. FTIR spectra of the iron oxide nanoparticles at different time points in the reaction cycle. Three and four time points are shown for the addition of a) 0.4 b) 0.8 equivalents of EDC, respectively. Broad $\mathrm{O}-\mathrm{H}$ band at $3000-3500 \mathrm{~cm}^{-1}$, acid functionalized iron oxide nanoparticles alkyl peaks at $2900 \mathrm{~cm}^{-1}\left(\mathrm{C}-\mathrm{H}_{2}\right.$ and $\left.\mathrm{C}-\mathrm{H}_{3}\right)$, carbonyl peak at $1705 \mathrm{~cm}^{-1}(\mathrm{C}=\mathrm{O})$. We observed additional NHS ester peaks appear, while the carbonyl peaks disappeared after EDC addition, at 1814,1784 , and $1738 \mathrm{~cm}^{-1}$ originating from the carbonyl groups of the NHS ester. In addition, characteristic signals of the succinimide cycle were observed at $1206 \mathrm{~cm}^{-1} \mathrm{vs}(\mathrm{C}-\mathrm{N}-\mathrm{C})$, and 1065 $\mathrm{cm}^{-1}$ vs(N-C-O). FTIR spectrum 24 hours or 3 days after EDC addition. The signals of NCs with acid surface groups have reappeared. 


\section{References}

(1) Duan, H.; Kuang, M.; Wang, X.; Wang, Y. A.; Mao, H.; Nie, S., Reexamining the Effects of Particle Size and Surface Chemistry on the Magnetic Properties of Iron Oxide Nanocrystals: New Insights into Spin Disorder and Proton Relaxivity. The Journal of Physical Chemistry C 2008, 112 (22), 8127-8131.

(2) Yonezawa, T.; Kunitake, T., Practical preparation of anionic mercapto ligand-stabilized gold nanoparticles and their immobilization. Colloids and Surfaces a-Physicochemical and Engineering Aspects 1999, 149 (1-3), 193-199.

(3) Hinterwirth, H.; Kappel, S.; Waitz, T.; Prohaska, T.; Lindner, W.; Lämmerhofer, M., Quantifying Thiol Ligand Density of Self-Assembled Monolayers on Gold Nanoparticles by Inductively Coupled Plasma-Mass Spectrometry. ACS Nano 2013, 7 (2), 1129-1136.

(4) Murphy, C. J.; Buriak, J. M., Best Practices for the Reporting of Colloidal Inorganic Nanomaterials. Chemistry of Materials 2015, 27 (14), 4911-4913.

(5) Anderson, S. L.; Luber, E. J.; Olsen, B. C.; Buriak, J. M., Substance over Subjectivity: Moving beyond the Histogram. Chemistry of Materials 2016, 28 (17), 5973-5975. 